\title{
Colección de mamíferos de referencia y exhibición del Museo de Ciencias Naturales Federico Carlos Lehmann Valencia, Cali, Colombia
}

\author{
Tatiana Velásquez-Roa ${ }^{1,2 *(i)}$, Wilber Alfonso Triana Diosa2 (i) \\ 1 Maestría en Ciencias Biología, Facultad de Ciencias Naturales y Exactas, Universidad del Valle, Calle 13 \# 100 - 00, Cali, Valle \\ del Cauca, Colombia. \\ 2 Departamento de Zoología, Museo Departamental de Ciencias Naturales Federico Carlos Lehman Valencia, INCIVA, Avenida \\ Roosevelt \# 24 - 80, Cali, Valle del Cauca, Colombia. \\ * Correspondencia: velasquezrtatiana@gmail.com

\section{Resumen}

La Colección de Mamíferos asociada al Museo de Ciencias Naturales Federico Carlos Lehmann Valencia (IMCN), hace parte de las colecciones zoológicas de referencia científica del Instituto para la Investigación y la Preservación del Patrimonio Natural y Cultural del Valle del Cauca - INCIVA. La colección de mamíferos de referencia cuenta con 166 especímenes, pertenecientes a 8 órdenes, 25 familias, 57 géneros y 64 especies. Los especímenes proceden de ocho departamentos de Colombia y de un estado de Estados Unidos. Adicionalmente, el Museo de Ciencias Naturales cuenta con una colección de mamíferos de exhibición que incluye 155 especímenes, agrupados en 11 órdenes, 35 familias, 64 géneros y 80 especies. Dada la importancia ecológica que tienen en los ecosistemas que habitan, se resalta la representación en las colecciones de especies como Cebuella pygmaea, Cyclopes dorsalis, Megaptera novaeangliae, Puma concolor, Panthera onca, Tremarctos ornatus y Tapirus pinchaque.

Palabras clave: Colecciones biológicas, ecosistemas terrestres y marinos, ejemplares, mastozoología, mamíferos en Colombia.

\section{Abstract}

The mammal's collection of the Museum of Natural Science Federico Carlos Lehmann Valencia (IMCN) is part of the scientific reference zoological collection of the Institute for Research and Preservation of the Natural and Cultural Heritage of Valle del Cauca - INCIVA. The scientific reference mammal collection includes 166 specimens, belonging to 8 orders, 25 families, 57 genera and 64 species. The specimens are from 8 departments in Colombia and one state in the United States. The Museum of Natural Science additionally has a mammal collection for exhibition of 155 specimens, belonging to 11 orders, 35 families, 64 genera and 80 species. The representation in the collections of species such as Cebuella pygmaea, Cyclopes dorsalis, Megaptera novaeangliae, Puma concolor, Panthera onca, Tremarctos ornatus and Tapirus pinchaque is highlighted due to the ecological importance they have in the ecosystems that inhabit.

Key words: Biological collections, Colombian mammals, Mammalogy, Specimens, Terrestrial and marine ecosystems. 
La Colección de Mamíferos del Museo Departamental de Ciencias Naturales Federico Carlos Lehmann Valencia (IMCN) está ubicada en Cali - Valle del Cauca y su creación data de 1963. Actualmente, hace parte de las colecciones zoológicas de referencia científica del INCIVA Instituto para la Investigación y la Preservación del Patrimonio Natural y Cultural del Valle del Cauca (Figura 1).

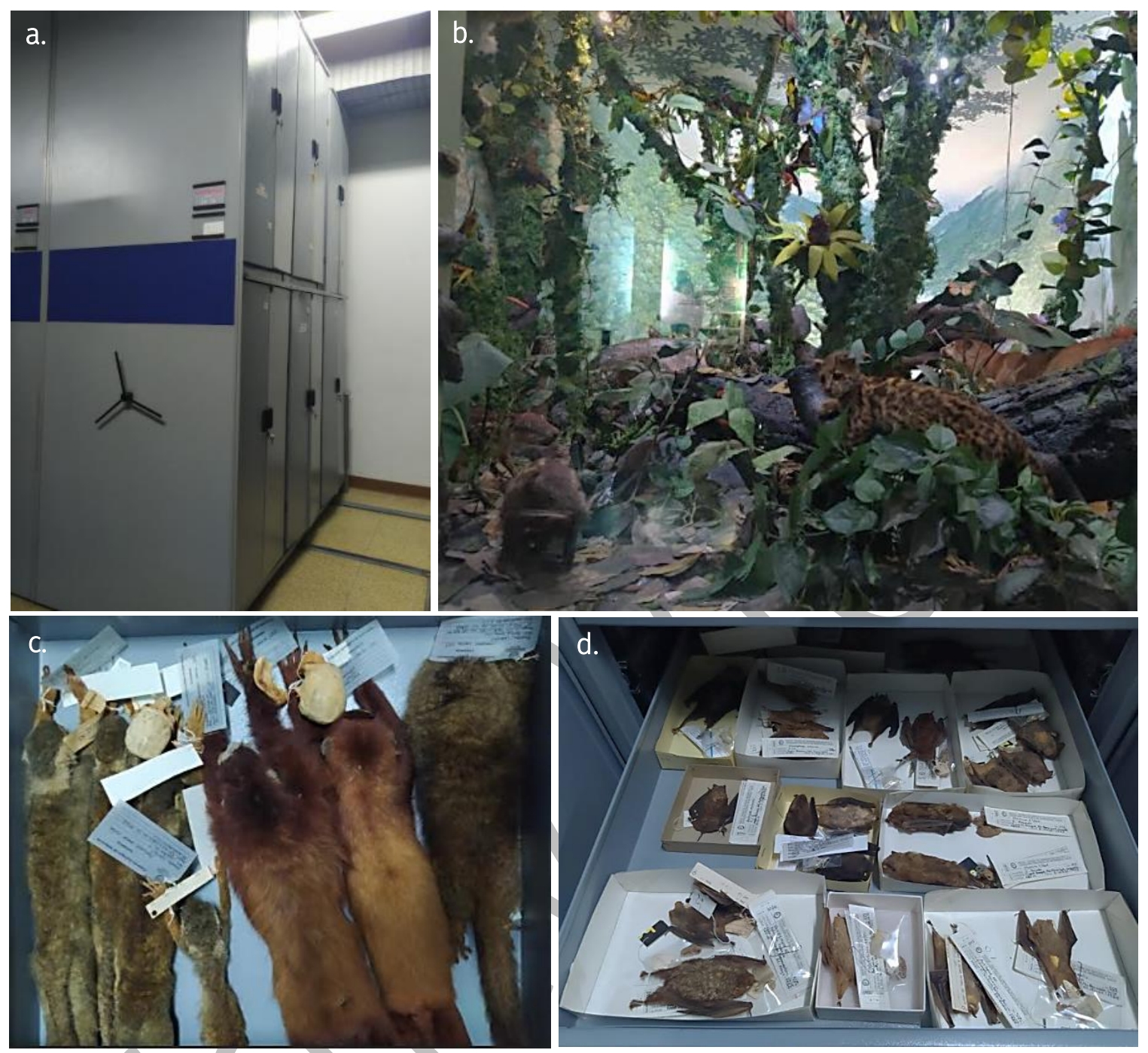

FIGURA 1. Colección de referencia científica y de exhibición del Museo de Ciencias Naturales Federico Carlos Lehmann. a. Almacenador de ejemplares de referencia científica. b. Dioramas con especímenes de exhibición. c - d. Especímenes de la colección de referencia científica IMCN.

La colección de referencia alberga 166 especímenes de mamíferos (conteo a 22 enero de 2021), pertenecientes a 8 órdenes, 25 familias (una exótica: Suidae), 57 géneros (uno exótico: Sus) y 64 especies (dos exóticas: Sus scrofa, Odocoileus hemionus). En total, 109 especimenes se encuentran catalogados y 57 están en proceso de catalogación. Los órdenes mejor representados son Chiroptera con 66 especimenes (31 especies), seguido por Rodentia con 25 especímenes (siete especies) (Tabla 1). Del total de especímenes, cinco se encuentran identificados a nivel de género (Didelphimorphia= 1 , Chiroptera $=4$ ) y sólo uno a nivel de familia. En cuanto a la cobertura geográfica, los especímenes de Colombia proceden de los departamentos de Amazonas (1), Caldas (1), Caquetá (6), Cauca (9), Meta 
(1), Putumayo (14), Valle del Cauca (132), y adicionalmente hay dos especímenes que provienen de California, Estados Unidos. En cuanto a la cobertura temporal, la colección alberga especímenes colectados desde 1953 hasta el 2020.

Por su parte, la colección de exhibición incluye 155 especímenes (conteo 29 enero de 2021), pertenecientes a 11 órdenes, 35 familias (tres exóticas: Camelidae, Hominidae y Muridae), 64 géneros (siete exóticos: Lama, Lycaon, Myocastor, Mus, Oryctolagus, Pongo y Rattus) y 80 especies (siete exóticas: Lama glama, Lycaon pictus, Myocastor coypus, Mus musculus, Oryctolagus cuniculus, Pongo pygmaeus, Rattus rattus). El orden con mayor representatividad es Carnivora con 47 especímenes (22 especies), seguido por Primates con 29 especímenes (18 especies), Artiodactyla (ocho especies) y Rodentia (nueve especies) con 21 especímenes cada uno. En el caso de la colección de mamíferos de exhibición del IMCN, es importante mencionar que incluye mamíferos característicos de diferentes ecosistemas terrestres y marinos presentes en el país tales como el páramo, bosque altoandino, bosque seco tropical, bosque pluvial, humedales y selvas bajas. En la Tabla 1 se incluye el número de familias, géneros y especies que reposan en la colección de exhibición.

TABLA 1. Órdenes, familias, géneros y especies de mamíferos depositados en la colección de Mamíferos de referencia y de exhibición del Museo de Ciencias Naturales Federico Carlos Lehmann Valencia (IMCN). NuE: Número de especímenes.

\begin{tabular}{lcccccccc}
\hline & \multicolumn{3}{c}{ Colección de Referencia } & \multicolumn{4}{c}{ Colección de Exhibición } \\
\cline { 2 - 9 } \multicolumn{1}{c}{ Órdenes } & Familias & Géneros & Especies & NuE & Familias & Géneros & Especies & NuE \\
Didelphimorphia & 1 & 3 & 3 & 4 & 1 & 3 & 3 & 6 \\
Paucituberculata & 1 & 1 & 1 & 2 & - & - & - & - \\
Cingulata & - & - & - & - & 1 & 2 & 2 & 3 \\
Pilosa & 2 & 2 & 2 & 8 & 4 & 5 & 6 & 12 \\
Chiroptera & 4 & 25 & 31 & 90 & 2 & 4 & 6 & 8 \\
Carnivora & 5 & 9 & 9 & 14 & 6 & 16 & 22 & 47 \\
Perissodactyla & - & - & - & - & 2 & 2 & 3 & 5 \\
Artiodactyla & 3 & 5 & 5 & 13 & 3 & 6 & 8 & 21 \\
Cetacea & - & - & - & - & 1 & 1 & 1 & 1 \\
Primates & 4 & 6 & 6 & 10 & 6 & 14 & 18 & 29 \\
Rodentia & 5 & 6 & 7 & 25 & 8 & 9 & 9 & 21 \\
Lagomorpha & - & - & - & - & 1 & 2 & 2 & 2 \\
\multicolumn{1}{c}{ Total } & 25 & 57 & 64 & 166 & 35 & 64 & 80 & 155 \\
\hline
\end{tabular}

La colección de mamíferos del IMCN ha sido vistada por un sinnúmero de investigadores con el fin de recopilar información. Los especímenes de la colección de mamíferos del IMCN han sido utilizados en al menos ocho investigaciones (Lehmann 1967; Alberico 1981, 1983; Velasco Abad \& Alberico 1984; Riascos-Vallejo 2001; Ramírez-Chaves \& Pérez $2010 a$, 2010b; Arenas \& Giraldo 2013).

Entre los investigadores que aportaron al crecimiento y consolidación de la colección de Mamíferos del IMCN se resalta a su fundador, Federico Carlos Lehmann Valencia, así como Eduardo Velasco Abad. Actualmente, el INCIVA está desarrollando un proyecto en pro de la conservación del Bosque Seco Tropical del Centro - Norte del Valle del Cauca, del cual a la fecha se han depositado alrededor de 60 especímenes en el IMCN y se espera que este número aumente en el momento en que se complete satisfactoriamente el proyecto. 


\section{AGRADECIMIENTOS}

Agradecemos a Andrea Bernal, Sara Medina y Pamela Carvajal por la colaboración en revisión del material existente en la colección de exhibición y especialmente a Cristián Calvache por su compromiso y colaboración en la preparación del material depositado en la colección y por sus comentarios a esta nota.

\section{REFERENCIAS}

Alberico M. 1981. Lista preliminar de los murciélagos del Valle del Cauca. Cespedesia 10 (39-40): 223230.

Alberico M. 1983. Lista anotada de los mamíferos del Valle del Cauca. Cespedesia 12 (45-46): 51-72.

Arenas D, Giraldo A. 2013. Quirópteros del Parque Natural Regional El Vínculo y su zona de amortiguación (Buga, Valle del Cauca, Colombia). Biota Colombiana 14 (3): 51-56. https:// doi.org/10.15472/ q6zitm

Lehmann VFC. 1967. Fauna de la laguna de Sonso. En: Llanos J, Patiño V. Editores. Proyecto de la Laguna de Sonso o del Chircal. Informe Técnico CVC No. 67-2. Santiago de Cali. Colombia. 13 p.

Ramírez-Chaves HE, Pérez WA. 2010a. Mamíferos (Mammalia: Theria) del departamento del Cauca, Colombia. Biota Colombiana 11 (1-2): 141-171. http:/ / doi.org/10.15472/ pc8lru

Ramírez-Chaves HE, Pérez WA. 2010b. Breve reseña histórica de la investigación mastozoológica en el departamento del Cauca, Colombia. Revista novedades colombianas 10 (1): 71-87. https:/ / revistas.unicauca.edu.co/index.php/novedades/article/view/1178

Riascos Vallejo JM. 2001. Aves, mamíferos, reptiles y anfibios de la Colección Zoológica de referencia "IMCN" del Museo de Ciencias Naturales "Federico Carlos Lehmann V." Cespedesia 24 (7578): 95-152.

Velasco Abad E, Alberico M. 1984. Notas sobre algunos mamíferos nuevos de la fauna vallecaucana. Cespedesia 13 (49-50): 291-295.

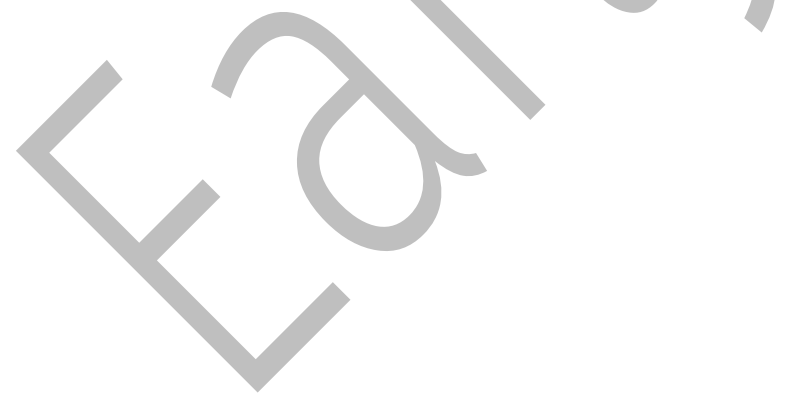

Editor: Héctor E. Ramírez-Chaves Recibido: 2021-01-30 Revisado:2021-02-01 Aceptado: 2021-02-08 Publicado: 2021-03-03 